\title{
Chromatic Dispersion Monitoring for High-Speed WDM Systems Using Two-Photon Absorption in a Semiconductor Microcavity
}

K. Bondarczuk, Member, IEEE, P. J. Maguire, Member, IEEE, D. Reid, L. P. Barry, Member, IEEE, J. O'Dowd, W. H. Guo, M. Lynch, A. L. Bradley, and J. F. Donegan, Member, IEEE

\begin{abstract}
This paper presents a theoretical and experimental investigation into the use of a two-photon absorption (TPA) photodetector for use in chromatic dispersion (CD) monitoring in highspeed, WDM network. In order to overcome the inefficiency associated with the nonlinear optical-to-electrical TPA process, a microcavity structure is employed. An interesting feature of such a solution is the fact that the microcavity enhances only a narrow wavelength range determined by device design and angle at which the signal enters the device. Thus, a single device can be used to monitor a number of different wavelength channels without the need for additional external filters. When using a nonlinear photodetector, the photocurrent generated for Gaussian pulses is inversely related to the pulsewidth. However, when using a microcavity structure, the cavity bandwidth also needs to be considered, as does the shape of the optical pulses incident on the device. Simulation results are presented for a variety of cavity bandwidths, pulse shapes and durations, and spacing between adjacent wavelength channels. These results are verified experimental using a microcavity with a bandwidth of $260 \mathrm{GHz}(2.1 \mathrm{~nm})$ at normal incident angle, with the incident signal comprising of two wavelength channels separated by $1.25 \mathrm{THz}(10 \mathrm{~nm})$, each operating at an aggregate data rate of $160 \mathrm{~Gb} / \mathrm{s}$. The results demonstrate the applicability of the presented technique to monitor accumulated dispersion fluctuations in a range of $3 \mathrm{ps} / \mathrm{nm}$ for $160 \mathrm{~Gb} / \mathrm{s} \mathrm{RZ}$ data channel.
\end{abstract}

Index Terms-Nonlinear detection, dispersive channels, wavelength division multiplexing (WDM), cavity resonators.

\section{INTRODUCTION}

D UE to continued growth of the Internet and the introduction of new broadband services, such as video-on-demand and mobile telephony, it is expected that individual channel data rates will exceed $100 \mathrm{~Gb} / \mathrm{s}$ in the next five years. One of the major limitations of operating at such high data rates over long

Manuscript received December 14, 2007; revised April 16, 2008. Current version published December 03, 2008. This work was supported in part by Enterprise Ireland's Commercialisation Fund Technology Development Research Programme (CFTD/2005/IT/303a,b) and by Science Foundation Ireland under its CTVR CSET Programme.

K. Bondarczuk , P. J. Maguire, D. Reid, and L. P. Barry are with RINCE, School of Electronic Engineering, Dublin City University, Dublin 9, Ireland (e-mail: krzysztof@eeng.dcu.ie; maguirep@eeng.dcu.ie; douglas.reid@eeng.dcu.ie; Liam.barry@dcu.ie).

J. O'Dowd, W. H. Guo, M. Lynch, A. L. Bradley, and J. F. Donegan are with Semiconductor Photonics Group, School of Physics and Centre for Telecommunication Value Driven Research (CTVR), Trinity College, Dublin 9, Ireland (e-mail: jodowd@tcd.ie; guow@tcd.ie; lynchmi@tcd.ie; bradlel@tcd.ie; jdonegan@tcd.ie)

Digital Object Identifier 10.1109/JQE.2008.2001942 distances will be the amount of chromatic dispersion (CD) that is introduced during pulse propagation. A number of different schemes, such as pre-chirp [1] and utilizing a modulation format with a narrow optical spectrum (duobinary [2]), have been introduced to improve system tolerance to CD. However, even by employing such techniques, there still exists a need to compensate for the amount of accumulated dispersion encountered within the telecommunications link. A commonly used technique, called dispersion management, involves including in the transmission path a fixed-value, negative-dispersion elements that compensate for the positive dispersion encountered by the pulse propagating in standard single mode fiber. A vast number of deployed systems use dispersion compensating fibers (DCF) although chirped fiber Bragg gratings (FBG) can also be used [3]. This technique however does not compensate for the additional CD fluctuations that arise due to variations of temperature [4], [5] or mechanical stress. Such variations will become a serious problem when individual channel data rates start to exceed $40 \mathrm{~Gb} / \mathrm{s}$. As such, it is important to develop new CD monitoring and tunable equalization techniques that have the ability to operate at such high data rates.

Currently a number of schemes have been proposed to compensate for chromatic dispersion utilizing a single FBG [6] or two nonlinearly chirped FBG used in tandem to mitigate third order dispersion effects [7]-[9]. Various monitoring techniques have been demonstrated such as adding a subcarrier tone to the transmitted data signal [10] or employing nonlinear detection of optical pulse distortions [11]. In this paper a technique based on the optical nonlinearity of two-photon absorption (TPA) in a specially designed microcavity is utilized for high-speed, wavelength selective, sequential CD monitoring of two wavelength division multiplexing (WDM) channels. Previous work has shown CD monitoring of $40 \mathrm{~Gb} / \mathrm{s}$ and $80 \mathrm{~Gb} / \mathrm{s}$ return-to-zero (RZ) signals in a single-wavelength channel system [12]. Here, device wavelength selectivity performance is investigated by using a two-wavelength channel system. Furthermore, the advantage of the resonance microcavity tuning by varying the incident angle is employed to demonstrate the sequential CD monitoring of two WDM channels. The experimental results are discussed utilizing a theoretical model including the effects of pulse overlapping and finite cavity bandwidth.

This paper is divided up as follows: In Section II the nonlinearity of TPA and the advantages of employing a microcavity design for the detector is reviewed. In Section III the TPA microcavities employed in the experimental setup is described and 

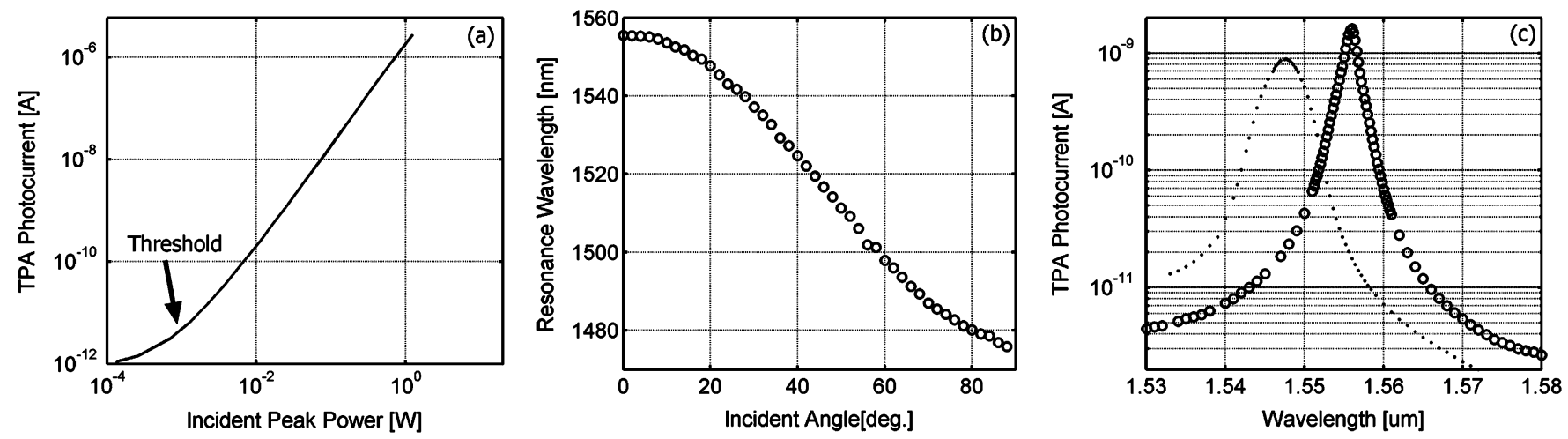

Fig. 1. (a) TPA photocurrent for varied incident pulse-signal average power; (b) measured cavity peak-resonance wavelength for varied incidence angle; (c) resonance characteristics for normal (circles) and $22^{\circ}$ (dots) incident angles.

characterized. In Section IV a theoretical model utilized to calculate the TPA microcavity nonlinear response is presented. In Section $\mathrm{V}$ the influence of the incident pulse shape profile on the cavity response is discussed. An experimental demonstration of CD monitoring in a multi-wavelength channel system is described in Section VI. In particular, the results presented highlight how the channel spacing and cavity bandwidth determine the amount of interference introduced by adjacent channels. In Section VII the TPA microcavity performance to monitor CD in a hybrid WDM/OTDM configuration is demonstrated, with a summary of the main experimental and theoretical work presented in this paper given in Section VIII.

\section{TPA MICROCAVITY}

Two photon absorption (TPA) is a nonlinear optical-to-electrical conversion process that occurs in semiconductors when two photons are simultaneously absorbed to generate a single electron-hole pair [13]-[16]. The semiconductor bandgap energy is chosen to be higher then the incident photon energy. Under this condition single photons do not possess sufficient energy to generate an electron-hole pair. Therefore, the majority of the photocurrent generated by the detector will result from TPA. This TPA photocurrent is proportional to the square of incidence optical power falling on the detector, and it is this nonlinear response, combined with TPA's ultra-fast response time, which enables TPA to be considered for high-speed CD monitoring.

One of the major problems associated with using the TPA process is the inherent inefficiency associated with the nonlinear process, resulting in the need for high optical intensities typically not found in optical communications networks. One way to overcome this problem is by the adoption of resonance cavity enhancement (RCE) technology within the detector. It has already been shown that by employing RCE technology, the TPA response can be enhanced by over four orders of magnitude when compared to non-cavity devices [17]. One interesting feature of using a RCE-based device is the fact that the incident signal is only enhanced over a narrow wavelength range determined by the device design. This characteristic of the microcavity allows the monitoring of a single WDM channel without the necessity of using an additional external optical filter. Such a device has already been employed for optical sampling applications [18]. Furthermore, the resonance peak of the cavity can be easily tuned by tilting the device, giving a possibility of sequentially monitoring different WDM channels using a single device [19].

It should be noted that the TPA process occurring in GaAs material is polarization sensitive [20]. This polarization dependence is further intensified by the employment of a resonance cavity structure. The above polarization effects are not included in this paper. In the theoretical and experimental investigation it was assumed that the polarization is fixed (set to maximise the generated TPA photocurrent).

\section{MiCROCAVITY CHARACTERIZATION}

In this paper, a TPA microcavity was employed for CD monitoring. The fabricated device consists of two GaAs-AlAs Distributed Bragg Reflector (DBR) mirrors surrounding an undoped $\mathrm{GaAs}$ active region. The active region is $460 \mathrm{~nm}$ thick with bandgap energy of $1.428 \mathrm{eV}$. The mirrors consist of altering quarter-wave AlAs and GaAs layers, with the top p-doped mirror consisting of 13 periods and the bottom n-doped mirror consisting of 23 periods. The device length is designed to enhance the TPA absorption wavelength within the $1.5-\mu \mathrm{m}$ wavelength range. Prior to carrying out any experimental work, some initial characterization of the TPA microcavity was carried out. Fig. 1 (a) shows a plot of the TPA photocurrent generated as a function of optical peak power for an incident $10 \mathrm{GHz}, 2$ ps pulse train. As shown in Fig. 1(a), the nonlinear response is visible over 3 orders of magnitude dynamic range, limited by Single Photon Absorption (SPA) at low incident powers.

As mentioned in Section II, one of the advantages of using a RCE design is the fact that the incident signal is only enhanced over a narrow spectral range determined by the microcavity design. It has already been shown that the resonance wavelength of the microcavity can be varied by changing the angle at which the incident signal enters the device [19]. Fig. 1(b) shows a plot of the resonance wavelength of the microcavity as a function of varying incident angle $\theta$. The incident signal was generated using a narrow-bandwidth, wavelength tunable continuous-waveform $(\mathrm{CW})$ source with the microcavity mounted on 
a rotation stage which allowed for precise adjustment of the incident angle. For each angle, the incident wavelength was tuned over the wavelength range $1.48-1.58 \mu \mathrm{m}$ in order to find a maximum cavity resonance response. The photocurrent generated was measured using a PicoAmmeter as a function of this incident wavelength. From the plot, the resonance wavelength can be tuned down by $10 \mathrm{THz}(80 \mathrm{~nm})$ by rotating the microcavity by $88^{\circ}$, with the device having a $\sin ^{2}(\theta)$ response [19]. Fig. 1(c) shows the TPA microcavity resonance characteristics for normal (circles) and $22^{\circ}$ (dots) incident angles, with $22^{\circ}$ of rotation corresponding to a resonance shift of $1.25 \mathrm{THz}(10 \mathrm{~nm})$. These angles will be used further in the experimental work described in later sections. The same $\mathrm{CW}$ source that was employed in the resonance wavelength tuning characterization was used here. The spectral linewidths measured at the $3 \mathrm{~dB}$ point are $260 \mathrm{GHz}$ $(2.1 \mathrm{~nm})$ and $600 \mathrm{GHz}(4.8 \mathrm{~nm})$ for normal and angled device respectively. The change in spectral linewidth for the angled device is due to the increased effective cavity length, and consequently different cavity finesse. The TPA photocurrent at peak wavelength resonance is 3-orders of magnitude greater when compared to the photocurrent generated for off-resonance wavelengths. This ratio would be further increased if higher incident optical power were used. However, as the optical power used was $5 \mathrm{dBm}$ (maximum output power of CW source,) the SPA process is not negligible in the off-resonance wings, which has the effect of changing the resonance characteristic width. This incident optical power influence on the resonance characteristic will be discussed in more detail in the theoretical section.

\section{TheORETICAL MODEL DESCRIBING TPA BASED CHROMATIC DISPERSION MONITORING}

In this section a theoretical investigation into the use of a TPA-based CD monitoring will be given for a Return-to-Zero (RZ) data signal. A finite cavity bandwidth will be included to simulate the cavity's response for wide bandwidth incident signals. One area of analysis will be concerned with the limitation resulting from temporal pulse overlapping arising from high levels of pulse broadening due to $\mathrm{CD}$.

According to the average response of any second-order nonlinear detector, the photocurrent generated for incident periodic sequence of pulses can be given by [11]

$$
I_{\mathrm{TPA}}^{\mathrm{Av}} \propto \frac{P_{\mathrm{ave}}^{2}}{D} \text { where } D=\frac{\langle P(t)\rangle^{2}}{\left\langle P^{2}(t)\right\rangle}=\eta \frac{\tau}{T} .
$$

$P_{\text {ave }}, P(t)$ and $D$ are the average power, time-varying power envelope and generalized duty cycle of the incident optical signal. The use of the brackets \langle\rangle in (1) refers to the fact that the power envelope has been time averaged, while $\tau$ and $T$ are the incident pulsewidth and the period of the signal, and $\eta$ is a weight factor associated with the incident pulse shape. When a periodic sequence of optical pulses propagates through a dispersive medium, such as an optical fiber, the duty cycle of the signal increases. Therefore, according to the above relationship, the measurement of an average TPA photocurrent can be employed for CD monitoring of ultra-fast signals without the use of fast electronics. In order to simplify the theoretical investigation presented here, optical pulses with a Gaussian pulse shape were employed. This allows us to take advantage of the fact that when Gaussian pulses propagate through a dispersive medium they maintain their Gaussian shape (weight factor $\eta$ does not change). Therefore, the photocurrent generated, given by (1), for Gaussian incident pulses can be simplified to an inverse incident pulsewidth relationship [12].

One side effect of employing a nonlinear technique is that the monitoring range is limited by pulse overlapping when the duration of the optical pulse $(\tau)$ of a periodic sequence of RZ pulses approaches the width of the bit slot (T). However, the technique presented here is ideally suited for small CD fluctuations that arise due to variation of temperature and mechanical stress. The initial theoretical investigation and experimental verification is carried out for $10 \mathrm{GHz}$ RZ pulses of approximately 2 ps duration, resulting in a duty cycle much lower than that commonly found in telecommunication networks. Consequently the pulse overlapping occurred for higher amounts of dispersion thus the monitoring range was much longer then it would be in a real case. This idealistic approach was taken to verify the accuracy of the proposed theoretical model before applying it to a high data-rate system. In Section VII the cavity performance is tested by employing the same 2 ps pulses modulated and multiplexed up to $160 \mathrm{~Gb} / \mathrm{s}$ to obtain realistic high data-rate operating conditions, with a duty cycle of approximately $30 \%$.

According to Section II, the nonlinear process can be enhanced by using a resonance microcavity structure within the detector. In order to investigate how such a structure will affect the TPA response for CD monitoring the following theoretical model was used. The initial optical signal was assumed to be a transform-limited Gaussian pulse. The spectral amplitude of this pulse was calculated utilising a Fourier transform with a quadratic phase term added to simulate pulse propagation through various lengths of dispersive fiber. Next the signal spectral amplitude within the cavity (including frequency detuning between the incident signal and cavity resonance) was shaped by the cavity field transfer function $H(f)$. Finally an inverse Fourier transform was used to obtain the temporal field $\left(E_{C}(\mathrm{t})\right)$ of the signal within the cavity

$$
\begin{aligned}
& E_{C}(t)=F^{-1}\{H(f-\Delta f) \cdot F\{E(t)\} \\
&\left.\cdot \exp \left(\frac{i(2 \pi f)^{2} \beta_{2} L}{2}\right)\right\}
\end{aligned}
$$

where $F\{\cdot\}, F^{-1}\{\cdot\}$ are the Fourier transform and the inverse Fourier transform respectively, $f$ is the incident signal frequency, $\Delta f$ is the signal detuning from the cavity resonance and $E(t)$ is the incident field. The exponential term in (2) describes the propagation of the signal through a fiber length $L$ with a group velocity dispersion $\beta_{2}$. The cavity field transform function $H(f)$ can be approximated by [21]

$$
H(f)=\frac{1}{1+\frac{R}{1-R}\left(1-\exp \left(i \frac{2 \pi f}{\mathrm{FSR}}\right)\right)}
$$

where FSR and $R$ are the TPA microcavity free spectral range and the geometric average of the top and bottom mirror reflectivity. The average TPA photocurrent generated within the mi- 
crocavity is a combination of SPA and TPA processes, and can be expressed as

$$
I_{\mathrm{TPA}}^{\mathrm{Av}}=\left\langle C_{\mathrm{TPA}}\left|E_{C}(t)\right|^{4}+C_{\mathrm{SPA}}\left|E_{C}(t)\right|^{2}\right\rangle
$$

where $C_{\mathrm{TPA}}, C_{\mathrm{SPA}}$ are the collections of constants including TPA and SPA coefficients respectively. The TPA response for the incident monochromatic $\mathrm{CW}$ light will simplify to:

$$
I_{\mathrm{TPA}}^{A v, f_{0}}=C_{\mathrm{TPA}}\left|E_{0} H\left(f_{0}\right)\right|^{4}+C_{\mathrm{SPA}}\left|E_{0} H\left(f_{0}\right)\right|^{2}
$$

where $E_{0}, f_{0}$ are the amplitude and the frequency of the incident optical signal. The constants $C_{\mathrm{TPA}}$ and $C_{\mathrm{SPA}}$ can be estimated from the measured PI curve shown in Fig. 1(a). The parameters $R$ and FSR of the resonance microcavity can be found by substituting (3) into (5) and comparing the theoretical plot of the average TPA photocurrent with the experimental results presented in Fig. 1(c). Fig. 2 shows the cavity resonance characteristics measured experimentally (dots) and the fitted theoretical lines (solid) for (a) normal and (b) $22^{\circ}$ incident angles. For easier analysis the cavity resonance frequency was shifted to zero and normalized to unity. A good fit between the theoretical lines and the measured results are maintained over two orders of magnitude of the TPA response. It should be noted that according to (5) the device resonance characteristic will differ depending on the optical power used for the device characterization. If the incident optical power was set below the SPA threshold [highlighted in Fig. 1(a)] the generated photocurrent would be clearly due to the SPA process over the whole spectral-range. The measured resonance characteristic would be then proportional to $|H(f)|^{2}$. Conversely for sufficiently high incident optical power, the TPA process would be dominant over the whole spectral-range resulting in a characteristic proportional to $|H(f)|^{4}$. The resonance characteristics shown in Fig. 1(c) are intermediate cases where the nonlinear TPA process is dominant around the resonance peak and linear SPA becomes dominant in the off-resonance characteristic wings. This property of the TPA microcavity causes difficulty in defining the cavity bandwidth parameter since the measured resonance characteristic depends on the incident power. In this paper the cavity bandwidth will be understood as a spectral width of the resonance characteristic taken at the $3 \mathrm{~dB}$ point assuming the dominance of the TPA process over the whole FSR.

If the incident signal operates with an optical power high enough to ensure that the TPA effect dominants over the SPA, the second term in (4) can be neglected. Furthermore, if the incident signal bandwidth is small compared to the cavity bandwidth, the cavity effect can be neglected and (4) becomes equivalent to the (1). However when the incident pulse has a bandwidth that is greater than the cavity bandwidth (the incidence pulsewidth is shorter than the cavity lifetime) the cavity will narrow the incident signal in the spectral domain. This spectral filtering (or selective enhancement) causes two different effects depending on the amount of dispersion encountered by the signal before being incident on the cavity. In the case of a transform-limited incident pulse, due to the Fourier time-bandwidth limit, the cavity filtering will result in the temporal broadening
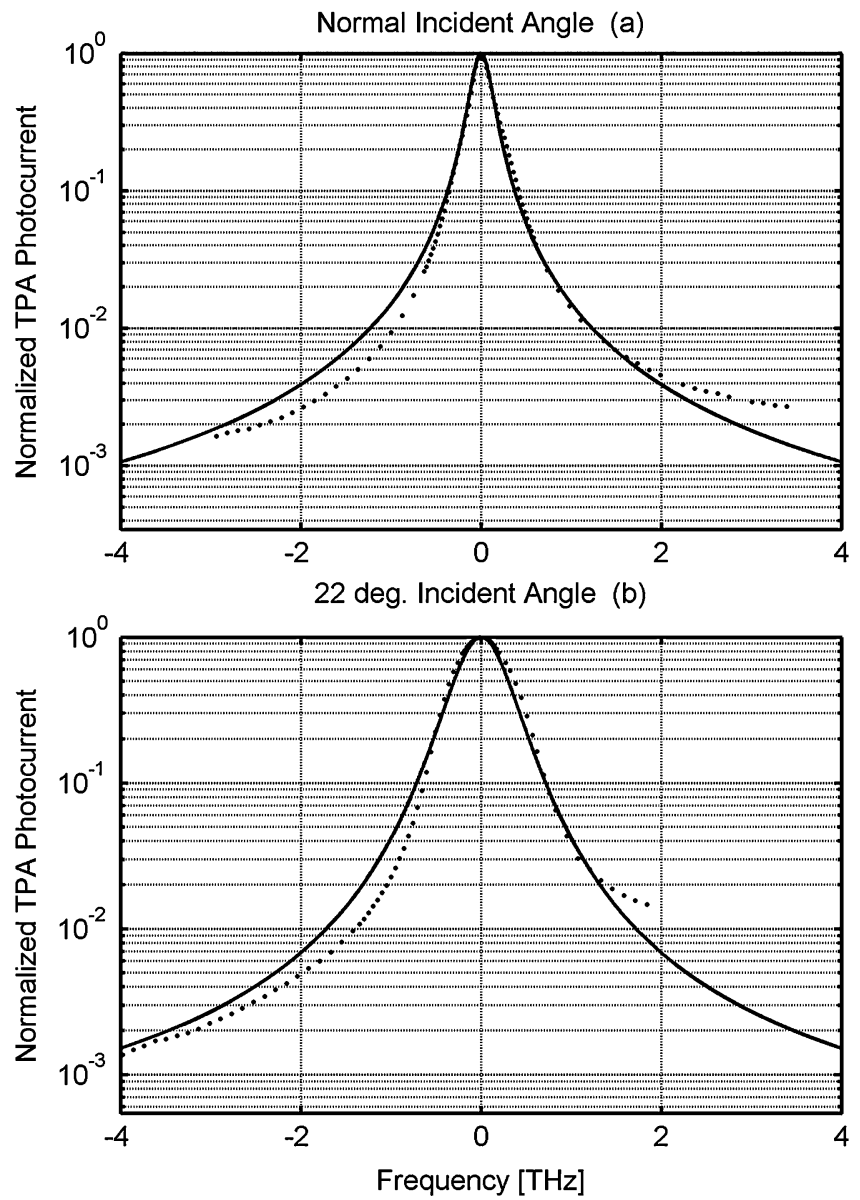

Fig. 2. Plot of the experimental (dots) and theoretical (solid line) TPA microcavity resonance responses for: (a) normal incident angle; (b) $22^{\circ}$ incident angle.

of the pulse within the cavity. However in the case of a dispersion-broadened pulse the fast spectral components of the pulse are shifted to the leading edge and the slow components remain in the trailing edge. The filtering of the signal removes from the spectrum the fastest and the slowest side spectral components responsible for the majority of dispersion broadening. Consequently the filtering results in a temporal narrowing of the pulse within the cavity. A detail mathematical description of this effect may be found in [22], [23]. Both effects lead to a certain inaccuracy of (1). To investigate how this effects the operation of a microcavity-based device for CD monitoring, a basic system simulation was carried out.

\section{A. Cavity Effect}

The simulation models a $10 \mathrm{GHz}$ RZ Gaussian incident periodic sequence, with a spectral width of $265 \mathrm{GHz}(2.1 \mathrm{~nm})$, corresponding to the characterized cavity bandwidth (at normal incident). The Fourier transform-limited pulse power envelope width was 1.66 ps. The cavity field and the TPA responses were calculated for various incident pulsewidths (resulting from the addition of a group delay component) according to (2) and (4). Fig. 3(a) plots the calculated generated photocurrents versus the incident pulsewidth for different cavity bandwidths varied around the incident signal spectral width. For easy comparison 
between different bandwidths, the cavity field $E_{C}$ was normalized to unity in the simulation and the SPA component was assumed to be negligible. As shown, when the cavity has a bandwidth of $500 \mathrm{GHz}(4 \mathrm{~nm})$, the cavity has little effect on dispersion monitoring performance, as the bandwidth is larger than the spectral width of the incident signal. The generated photocurrent is therefore inversely proportional to the incident pulsewidth and obeys (1). However, as the bandwidth of the cavity approaches the spectral width of the incident signal, the cavity effect results in the bending of the simulated curve [as shown in Fig. 3(a)]. The effect becomes more significant when the cavity bandwidth decreases. The simulation also includes the pulse overlapping effect leading to a flattening of the simulated curves. For a RZ pulse sequence the flattening occurs when the pulsewidth within the cavity reaches the length of a single period, which for a $10 \mathrm{GHz}$ pulse train is $100 \mathrm{ps}$. According to the previous discussion, a narrow bandwidth cavity compresses strongly dispersed pulses. Therefore, the flattening threshold occurs when longer incident pulsewidths are incident on narrower bandwidth cavity. According to Fig. 3(a) and with agreement to the theory for a $135 \mathrm{GHz}$ cavity bandwidth, the flattening occurs when the incident pulsewidth approach $200 \mathrm{ps}$.

In the simulation, the successive pulses were assumed to be mutually incoherent. This is a reasonable assumption in telecommunication systems where signals are propagated through long lengths of fiber, causing significant coherency degradation between successive pulses. However, if the coherence between successive pulses was maintained, the interference effects could not be neglected. The pulses would then combine coherently resulting in a Talbot (self-imaging) effect [24], [25]. CD monitoring assuming coherent pulse overlapping has been previously dealt with in [11]. The overall effect of this pulse (coherent or incoherent) overlapping will be to limit the range over which the $\mathrm{CD}$ of the signal can be monitored. Since the focus of this paper is on device performance over the monitoring range, pulse combining above the overlapping threshold will be assumed to be incoherent for simplicity. However in the experiments described later some residual pulse reconstruction was observed when the signal operated at a data rate of $160 \mathrm{~Gb} / \mathrm{s}$.

\section{B. Wavelength Selective Property of the Microcavity}

The same model as used in the cavity-effect simulation part was employed here to demonstrate the device wavelength selectivity for use in WDM systems. In the simulation, two Gaussian periodic sequences operating at different wavelengths were modelled. Both channels were operating at $10 \mathrm{GHz}$ repetition rates with channel spectral width of $265 \mathrm{GHz}(2.1 \mathrm{~nm})$ which coincides with the measured normal-incident cavity bandwidth of the device described in Section II. The initial transform limited pulsewidth was 1.66 ps. The first channel (denoted as the monitored channel) operated at the cavity resonance frequency $192.7 \mathrm{THz}(1556 \mathrm{~nm})$ and the second channel (denoted as the additional channel) was shifted from the cavity resonance by a varied spectral detuning. The cavity fields for both channels were calculated separately using (2) assuming $265 \mathrm{GHz}$ microcavity bandwidth and combined incoherently to obtain the cavity field $E_{C}$. The average photocurrent was
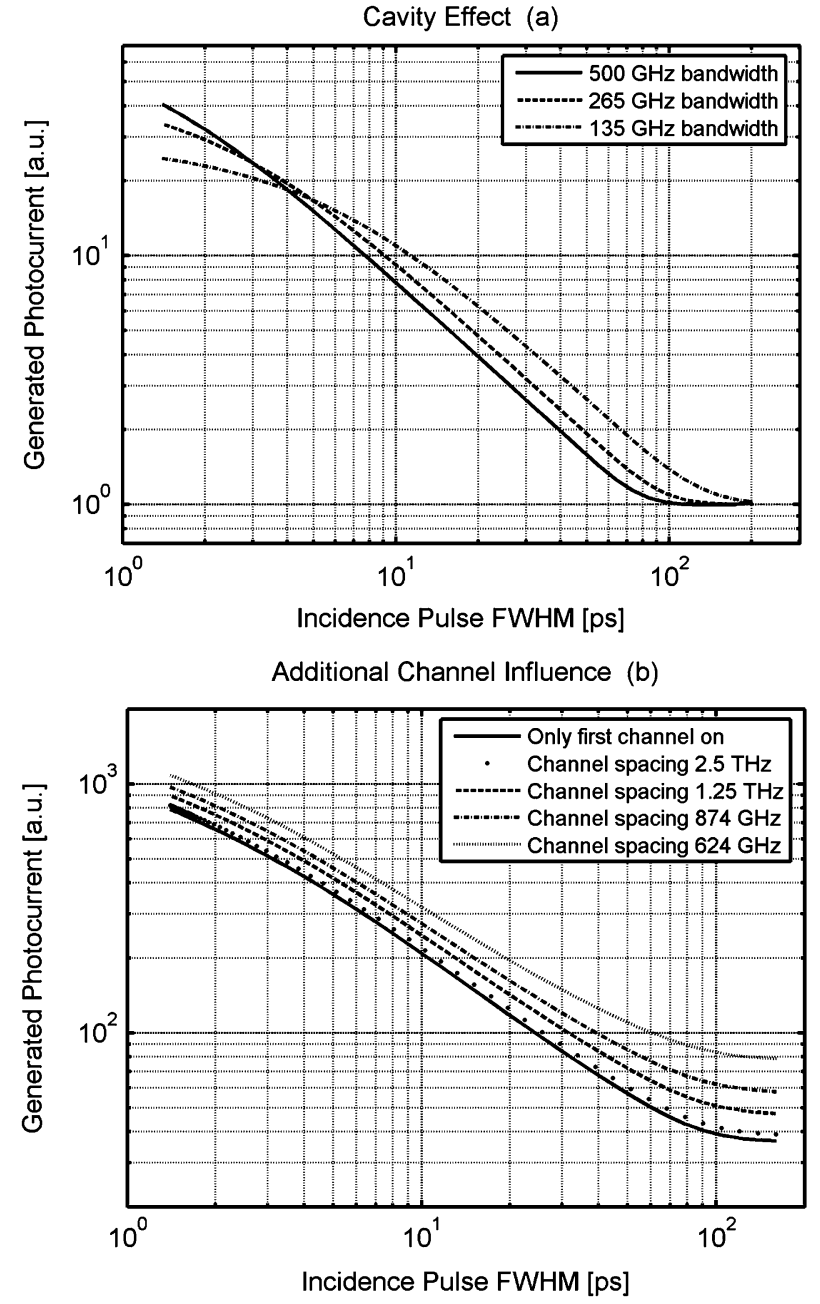

Fig. 3. Theoretical plots of the TPA photocurrent generated versus incident pulsewidth (FWHM) of a $10 \mathrm{GHz}$ Gaussian pulse train operating at the cavity resonance wavelength. (a) Response for varied cavity bandwidths showing the cavity effect. (b) Response for $265 \mathrm{GHz}$ cavity bandwidth influenced by additional channel for varied frequency spacing.

calculated utilizing (4) assuming that the TPA process dominants over SPA. Fig. 3(b) shows a plot of calculated aggregate TPA photocurrent versus the monitored channel pulsewidth for different wavelength channel separation. The pulses from the additional channel were always transform-limited and overlapped temporarily with the pulses from the monitored channel to consider the worst case scenario. From the results, the additional channel would lead to a slight overestimate of pulsewidth. According to Fig. 3(b), for a channel separation of $2.5 \mathrm{THz}(20 \mathrm{~nm})$ the influence from the additional channel is nearly negligible (less than $4 \%$ difference in photocurrent) while for $1.25 \mathrm{THz}(10 \mathrm{~nm})$ separation, the influence is slightly below $25 \%$.

According to the presented simulation results, the TPA microcavity can be used for selective wavelength channel CD monitoring. It is clear that the cavity bandwidth and the channel spacing play a significant role in determining the optimum performance of the CD monitoring. Thus, it is important to design the microcavity's bandwidth based on the channel bandwidth and the channel separation employed in the multi-wave- 
length system. From the results shown in Fig. 3(b), it can be seen that for the current device design, the level of interference between adjacent channels would be unacceptable for channel separation commonly used in multi-wavelength systems (3 times channel bandwidth). The channel spacing may be reduced by decreasing the cavity bandwidth, which would also increase the cavity response. By altering the cavity design it may be feasible to achieve a more optimal cavity resonance profile which would allow for further reduction in the channel separation. In this paper, however, due to the current device limitations the analysis of the cavity wavelength selectivity will be carried out for higher channel separations than used in typical optical communications networks. We will also assume that the variation in power level for the different channels falling on the detector is negligible.

The analysis carried out was confined to the case when the incident optical pulses had a Gaussian pulse shape. The response of the TPA microcavity to different pulse shapes will be discussed in the next section.

\section{Pulse Shape Influence ON THE Monitoring CHARACTERISTIC}

Since Gaussian pulses maintain their shape during propagation through a dispersive fiber, the TPA response given by (1) can be simplified to an inverse pulsewidth relationship for a wide bandwidth cavity. From Fig. 3(a) this inverse pulsewidth relationship holds true for a microcavity with a bandwidth of 500 GHz. However, when the cavity bandwidth approaches the incident signal bandwidth, the cavity effect causes the response to deviate from (1). This is shown in Fig. 3(a) for the devices with a bandwidth of $265 \mathrm{GHz}$ and $135 \mathrm{GHz}$. The above example illustrates the applicability of (1). It should be noted that the Gaussian pulse shape assumption is not always accurate in deployed systems. The pulses used in the experimental work presented in this paper are best described by sech ${ }^{2}$ power envelope. Since defining the pulsewidth is troublesome when the pulse shape is not maintained during pulse propagation, the modelled TPA photocurrents will be displayed as a function of Accumulated Dispersion (AD) instead of dispersed pulsewidth. AD is defined as the product of the fiber length and the fiber dispersion, and can be easily found independently of the pulse shape. The influence that the pulse shape has on the monitoring performance was investigated by comparing the response for a Gaussian pulse shape and a Sech ${ }^{2}$ pulse shape. The results of this are presented in Fig. 4(a)-(c). Fig. 4(a) and (b) show the modelled pulse power envelopes and spectral amplitudes for both pulse shapes. Both pulses had an initial Fourier transformlimited pulsewidth (FWHM) of $1.4 \mathrm{ps,} \mathrm{with} \mathrm{average} \mathrm{powers} \mathrm{nor-}$ malized. The pulses dispersion broadening and the nonlinear response were calculated using (2) and (4). Since the simulation was focused on the pulse shape influence on the nonlinear response, the cavity effects were neglected assuming infinite cavity bandwidth $(H(f)=1)$. Simulated TPA photocurrents versus introduced $\mathrm{AD}$ for both initial profiles are shown in Fig. 4(c). As shown, there is a slight difference between the amounts of photocurrent generated for the same amount of $\mathrm{AD}$ experienced by the two pulses. This can be accounted for by the fact that the different pulses evolve in a different manner while propagating through a fiber and within the microcavity. This simple result demonstrates that the CD characteristic depends on the pulse profile. The monitoring range is limited by the pulse flattening, which for a Gaussian pulse occurs at around $30 \mathrm{ps} / \mathrm{nm}$ and at $40 \mathrm{ps} / \mathrm{nm}$ for a $S e^{2}$ pulse.

\section{EXPERIMENTAL OF CHROMATIC DISPERSION MONITORING in a Multi-Channel CONFIGURATION}

According to Section II, the TPA microcavity can be employed for high bit rate, wavelength selective $\mathrm{CD}$ monitoring. In this section, the experimental CD monitoring of $10 \mathrm{~Gb} / \mathrm{s} \mathrm{RZ}$ signal is presented when an additional wavelength channel is included. The experimental setup is shown in Fig. 5(a). Both $10 \mathrm{GHz}$ RZ optical signals were generated using a wavelength tuneable (1450-1570 nm) actively mode-locked pulse sources. The generated sech ${ }^{2}$ pulses had a 1.4 ps transform limited temporal width and $312 \mathrm{GHz}(2.5 \mathrm{~nm})$ spectral bandwidth. The monitored channel was fixed at $192.7 \mathrm{THz}(1556 \mathrm{~nm}$ - resonance wavelength of the microcavity for normal incidence). Varied amounts of $\mathrm{AD}$ were introduced by passing the signal through different lengths of dispersion compensating fiber (DCF) ranging from 0 to $80 \mathrm{~m}$. The fiber parameters measured at $1550 \mathrm{~nm}$ were $\mathrm{D}=-99.3 \mathrm{ps} / \mathrm{km} . \mathrm{nm}$, Dispersion Slope $=-0.21 \mathrm{ps} / \mathrm{km} \cdot \mathrm{nm}^{2}$ and PMD $=0.18 \mathrm{ps} / \sqrt{ } \mathrm{km}$. The second channel was detuned spectrally from the first channel by $624 \mathrm{GHz}(5 \mathrm{~nm}), 874 \mathrm{GHz}(7 \mathrm{~nm})$ and $1.25 \mathrm{THz}(10 \mathrm{~nm})$. Both channels were combined together and incident on the TPA microcavity characterized in Section III. Erbium-doped fiber amplifiers (EDFAs) and inline power meters were employed to ensure desired power levels in both channels were maintained and to compensate for losses after propagation through the different lengths of DCF. The power levels and fiber lengths were chosen to avoid self-phase modulation and polarization mode dispersion effects. To ensure the same optical signal-to-noise ratio (OSNR) after amplification, the power level after each length of DCF (before the amplifier) was set at $-7 \mathrm{dBm}$. Both channels were incident at normal angle on the TPA microcavity, with the generated photocurrent measured using a highly sensitive PicoAmmeter. To investigate the worst possible scenario, pulses in the additional channel were nearly transform-limited and were overlapped temporarily with pulses in the monitored channel. Temporal alignment between the channels was controlled using an optical delay line (ODL). The measured TPA photocurrent generated versus introduced AD in the monitored channel for various spectral detuning of the additional channel is shown in Fig. 5(b).

As shown, the simulated response is in close agreement with the experimental results. The influence from the additional channel decreases with increased detuning between the channels. For channel separation of $1.25 \mathrm{THz}$, the influence is less then $25 \%$, which is in line with simulation results described earlier. According to the theoretical section a further increase in the channel separation would result in a negligible interference from the additional channel. Nevertheless the theoretical model presented here described correctly the cross-channel interference and may be employed to estimate the TPA microcavity response in multi-wavelength applications. The next section 

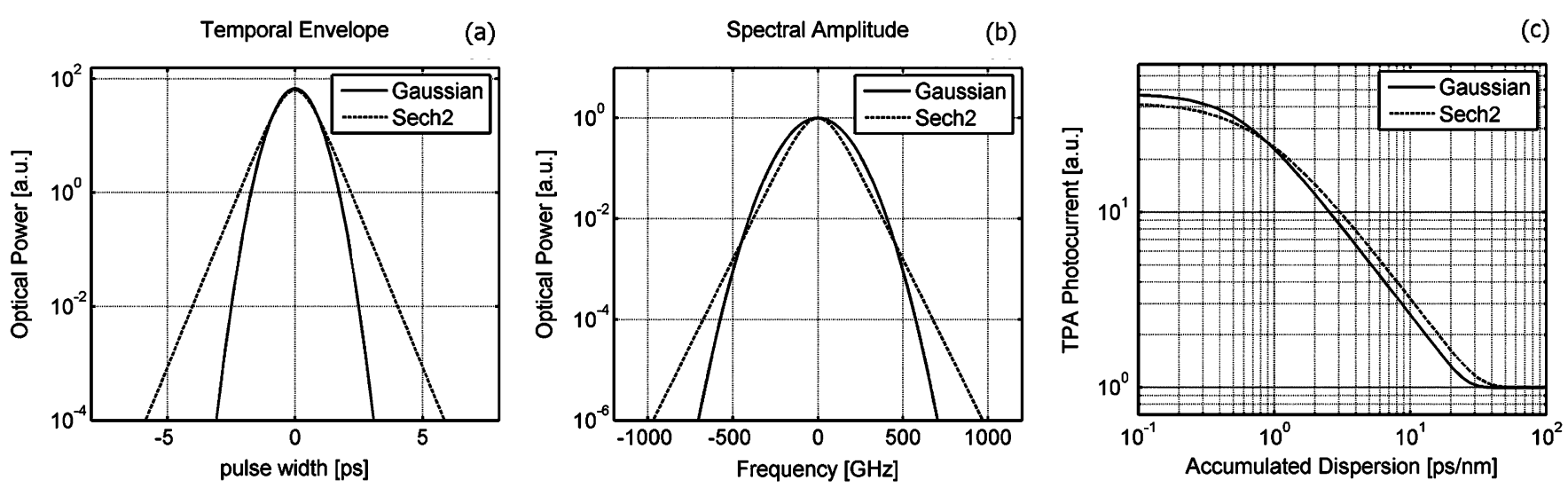

Fig. 4. Modelled (a) temporal power envelopes and (b) spectral amplitudes for Gaussian and Sech2 pulse shapes. (c) Theoretical plot of the TPA photocurrent versus Accumulated Dispersion introduced in the channel for Gaussian and Sech2 pulses.
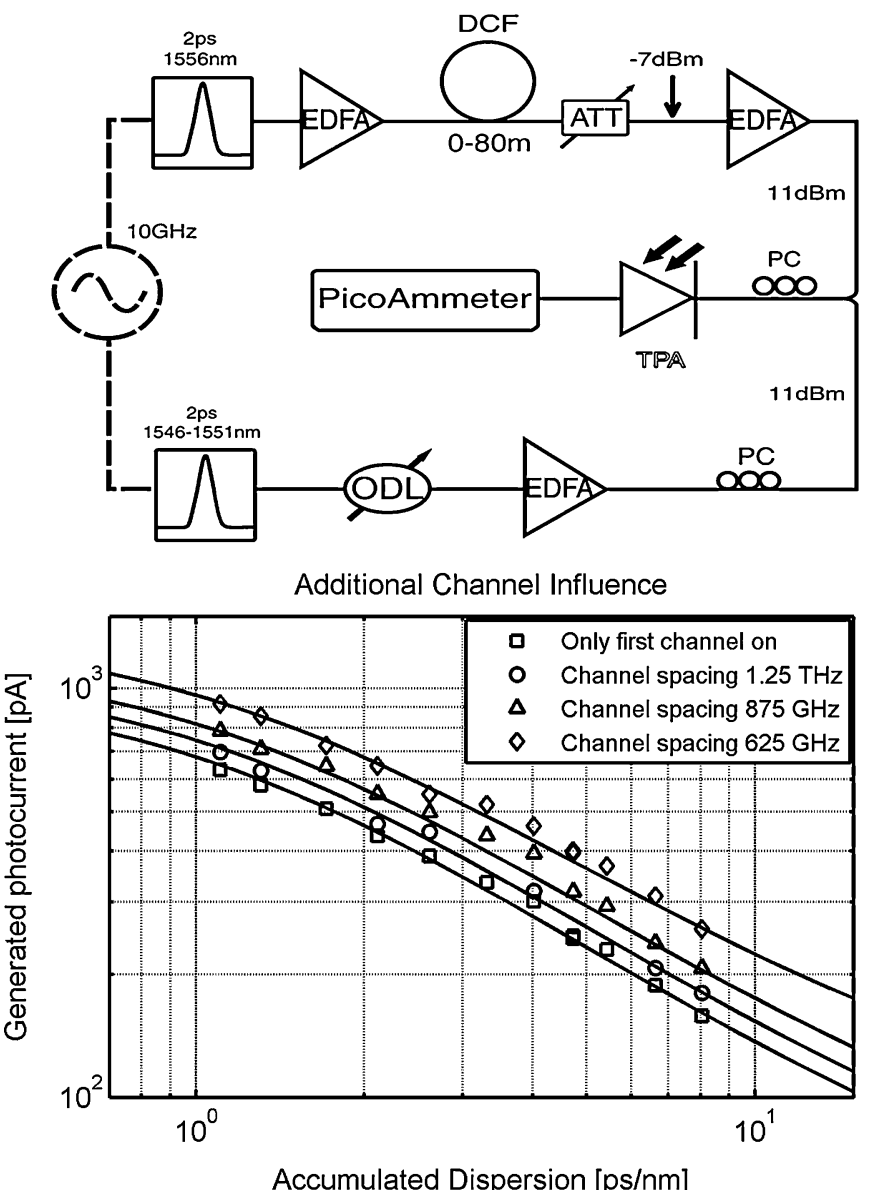

Fig. 5. (a) Experimental setup used to demonstrate $\mathrm{CD}$ monitoring in a multi-wavelength system; (b) Experimental results when the second channel was turned off (square) and when the second channel was on, detuned by 10 $\mathrm{nm}$ (circle), $7 \mathrm{~nm}$ (triangular) and $5 \mathrm{~nm}$ (diamond). Solid lines are simulated curves for the four separate scenarios.

describes the CD monitoring of two wavelength channels, each operating at $160 \mathrm{~Gb} / \mathrm{s}$, separated by $1.25 \mathrm{THz}(10 \mathrm{~nm})$.

\section{Selective Channel Chromatic Dispersion MONitoring at High Bit Data Rates}

It was previously mentioned that the cavity resonance can be altered by varying the angle of the incident signal. Here exper- imental work was undertaken to monitor the $\mathrm{CD}$ of two wavelength channels, each operating at an aggregate data rate of 160 $\mathrm{Gb} / \mathrm{s}$. The experimental setup is shown in Fig. 6. The same pulse sources described in previous sections were employed here. The wavelength channels were separated spectrally by $1.25 \mathrm{THz}$ $(10 \mathrm{~nm})$, operating at frequencies of $192.7 \mathrm{THz}(1556 \mathrm{~nm})$ and 193.9 THz (1546 nm). Both channels were amplified and combined together using a $3 \mathrm{~dB}$ passive fiber coupler. ODL's were used to overlap pulses temporally and to center them in the middle of the modulator window. The modulator was driven with a $2^{7}-1$ RZ pseudo-random bit sequence (PRBS) data pattern. Both channels were then passed through an OTDM multiplexer, consisting of four independently switch-able stages with fixed delay lengths within each stage. The multiplexer was followed by a polarizer to ensure the same polarization state in each OTDM channel (each stage included independent polarization control to maximize a power level after the polarizer). The WDM signal was then passed through different lengths of DCF described before. EDFA's and polarization controllers (PCs) were used to compensate for losses from the modulation and multiplexing stages, and to optimize the polarization states of the propagating signal. An optical attenuator (ATT) was placed after the various lengths of DCF to ensure that the optical power levels before the last EDFA were fixed, thereby ensuring the same OSNR. The average power level falling on the TPA microcavity was fixed at $6 \mathrm{dBm}$. The generated photocurrent for the various DCF lengths and data rates was measured on the PicoAmmeter. Initially, the cavity was set to normal incidence to monitor $\mathrm{CD}$ in the first channel. Then the cavity was angled by $22^{\circ}$ (to shift the cavity resonance wavelength by $10 \mathrm{~nm}$ ) to monitor the second channel.

The measured TPA photocurrents versus the introduced accumulated dispersion for both wavelength channels are displayed in Fig. 7. The measurements were carried out for 10, 40, and $160 \mathrm{~Gb} / \mathrm{s}$ channel data rates. The solid lines are simulation results obtained with the model described before, assuming incoherent pulse overlapping, different cavity bandwidths for normal and $22^{\circ}$ incident angles (Fig. 2), and the inclusion of interference of the additional wavelength channel. The flattening threshold can be clearly seen when operating at $160 \mathrm{~Gb} / \mathrm{s}$, with residual coherent interference between combined pulses 


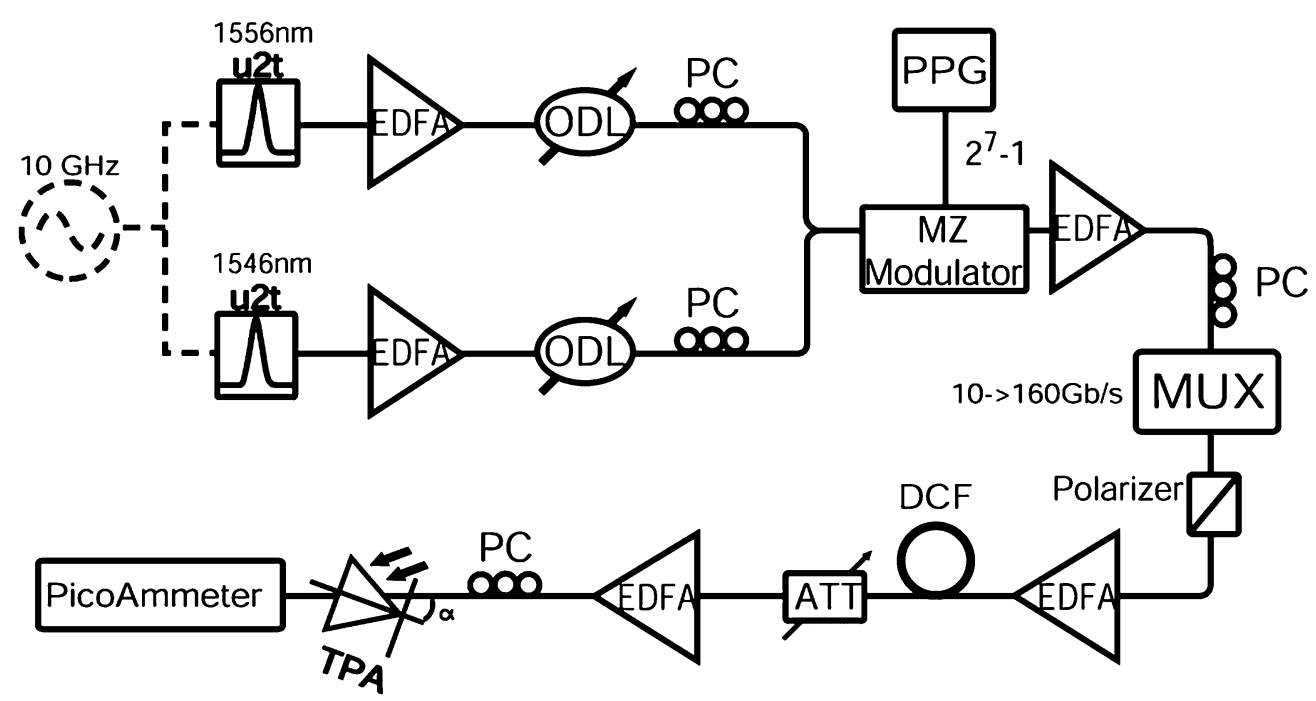

Fig. 6. Experimental setup used for sequential selective CD monitoring of two high-speed wavelength channels using a TPA microcavity.

leading to a small increase in the generated TPA photocurrent above $4 \mathrm{ps} / \mathrm{nm}$ for channel 1 and around $5 \mathrm{ps} / \mathrm{nm}$ for channel 2. The difference between the photocurrent generated for the two channels can be accounted for by the fact that channel 2 is incident on the TPA microcavity at an angle of $22^{\circ}$ off normal. This results in a different level of coupling and enhancement factor for that channels signal.

This section has experimentally demonstrated CD monitoring of two wavelength channels separated by $1.25 \mathrm{THz}(10 \mathrm{~nm})$ using a TPA microcavity. The microcavity had a normal-incident bandwidth of $265 \mathrm{GHz}$, with each wavelength channel operating at an aggregate data rate of $160 \mathrm{~Gb} / \mathrm{s}$. The level of interference caused by adjacent channels can be minimized by using a high finesse cavity designed for the desired WDM channel separation for the particular system being monitored.

\section{CONCLUSION}

In this paper, a theoretical and experimental investigation on the use of a TPA-based microcavity for CD monitoring in a highspeed, multi-wavelength optical communications system is presented. As mentioned, one of the major advantages of using a microcavity structure within the detector is that the signal is only enhanced over a narrow wavelength range, which is defined by the structure and design of the microcavity. In addition, by varying the angle of the incident signal, the resonance response peak of the device can be tuned, thereby isolating individual wavelength channels without the need for external optical filtering. A theoretical investigation into the suitability of using such a device was presented, with major findings including the fact the monitoring range is limited by pulse overlapping, which is further affected by the cavity bandwidth, and the shape of the optical pulses. In addition the wavelength spacing of the data channels is also an important parameter. These finding were then experimentally verified by carrying out the $\mathrm{CD}$ monitoring of two wavelength channels spaced by $1.25 \mathrm{THz}(10 \mathrm{~nm})$ using a microcavity that had a bandwidth of $260 \mathrm{GHz}(2.1 \mathrm{~nm})$ at normal incident angle. By rotating the device by $22^{\circ}$ it was possible to monitor the second channel. Both channels operated at an ag-
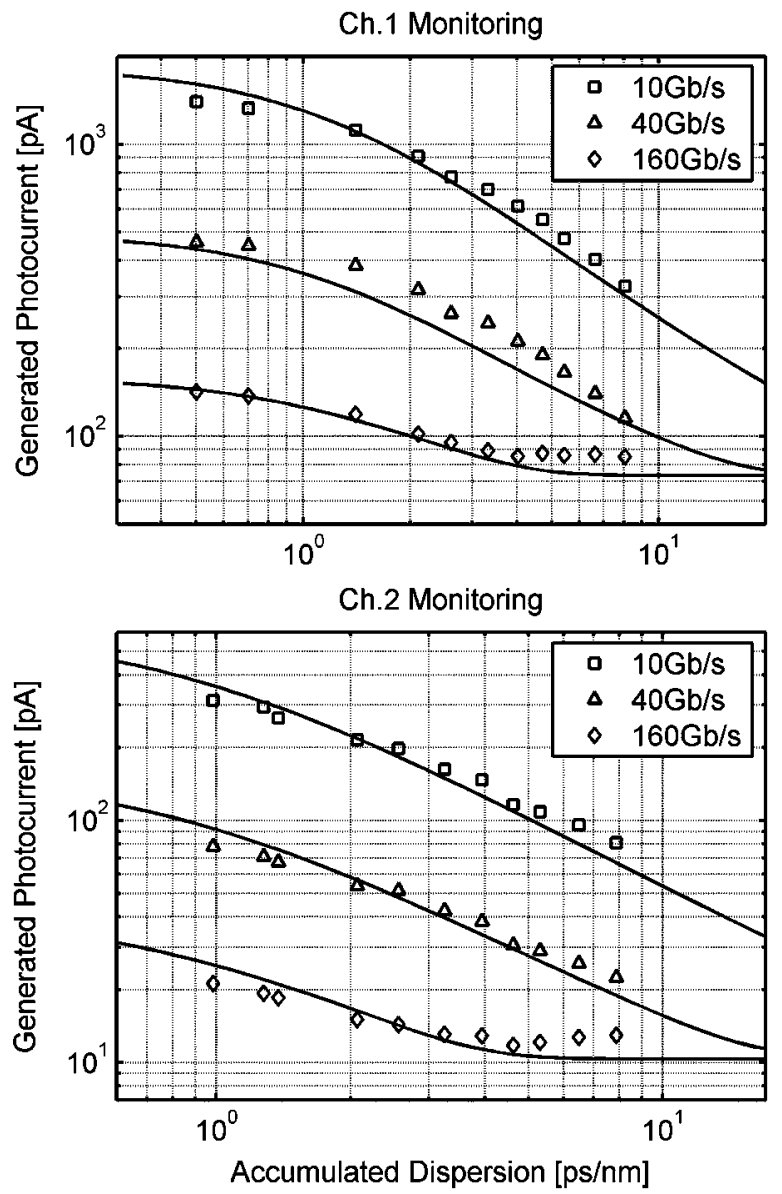

Fig. 7. Sequential CD monitoring of: (a) Channel 1 operating at $1556 \mathrm{~nm}$; (b) Channel 2 operating at $1546 \mathrm{~nm}$. The points correspond to the experimental results for various incident data rates, while the solid lines are the simulation curves assuming pulses incoherent overlapping and finite cavity bandwidth, including influence from additional channel spaced by $10 \mathrm{~nm}$.

gregate data rate of $160 \mathrm{~Gb} / \mathrm{s}$. The presented theoretical model assumes that all of the optical power is concentrated in the monitored signal which may not be accurate in real system measurements (due to OSNR limitations). In the experimental work 
the OSNR was kept above $20 \mathrm{~dB}$ allowing the model presented here to be applied to the experimental results obtained. Current work aims to expand on the work presented here to allow for the simultaneous measurement of OSNR and CD using nonlinear TPA detection. The presented results show the possibility of use the TPA microcavity device for detection and monitoring of additional CD fluctuations that may arise due to variations of temperature or mechanical stress.

Currently at $160 \mathrm{~Gb} / \mathrm{s}$ the dispersion can be determined up to $\sim 3 \mathrm{ps} / \mathrm{nm}$ with a much larger measurement range possible at lower bit rates. The maximum level of accumulated dispersion that can be measured at high repetition rates is limited by pulse overlap, while the minimum level is determined by a flattening of the current response around zero dispersion. However in all the cases presented here a maximization of the TPA photocurrent results in a minimization of accumulated dispersion.

\section{ACKNOWLEDGMENT}

The authors would like to thank Dr. D. Kilper of AlcatelLucent for helpful discussions.

\section{REFERENCES}

[1] T. Saito, N. Henmi, S. Fujita, M. Yamaguchi, and M. Shikada, "Prechirp technique for dispersion compensation for a high-speed long-span transmission," IEEE Photon. Technol. Lett., vol. 3, no. 1, pp. 74-76, Jan. 1991.

[2] X. Gu and L. C. Blank, "10 Gbit/s unrepeatered three-level optical transmission over $100 \mathrm{~km}$ of standard fiber," Electron. Lett., vol. 29, no. 25, pp. 2209-2211, 1993.

[3] M. J. Cole, K. Geiger, R. I. Laming, S. Y. Set, M. N. Zervas, W. H. Loh, and V. Gusmeroli, "Broadband dispersion-compensating chirped fibre Bragg gratings in a $10 \mathrm{Gbit} / \mathrm{s}$ NRZ $110 \mathrm{~km}$ non-dispersion-shifted fibre link operating at $1.55 \mu \mathrm{m}$," Electron. Lett., vol. 33, no. 1, pp. 70-71, 1997.

[4] W. H. Hatton and M. Nishimura, "Temperature dependence of chromatic dispersion in single mode fibers," J. Lightw. Technol., vol. LT-4, no. 10 , pp. $1552-1555$, Oct. 1986.

[5] T. Kato, Y. Koyano, and M. Nishimura, "Temperature dependence of chromatic dispersion in various types of optical fibers," Proc. OFC 2000, vol. 1, pp. 104-106, Paper TuG7.

[6] T. N. Nielsen, B. J. Eggleton, J. A. Rogers, P. S. Westbrook, P. B. Hansen, and T. A. Strasser, "Dynamic post dispersion optimization at $40 \mathrm{~Gb} / \mathrm{s}$ using a tunable fiber Bragg grating," IEEE Photon. Technol. Lett., vol. 12, no. 2, pp. 173-175, Feb. 2000.

[7] J. A. J. Fells, S. E. Kanellopoulos, P. J. Bennett, V. Baker, H. F. M. Priddle, W. S. Lee, A. J. Collar, C. B. Rogers, D. P. Goodchild, R. Feced, B. J. Pugh, S. J. Clements, and A. Hadjifotiou, "Twin fiber grating tunable dispersion compensator," IEEE Photon. Technol. Lett., vol. 13, no. 9, pp. 984-986, Sep. 2001.

[8] J.-X. Cai, K. Feng, A. Willner, V. Grubsky, D. Starodubov, and J. Feinberg, "Sampled nonlinearly-chirped FBG for tunable dispersion compensation of many WDM channels simultaneously," Proc. Optical Fiber Commun., 1999, Paper FA7.

[9] Z. Pan, Y. W. Song, C. Yu, Y. Wang, Q. Yu, J. Popelek, H. Li, Y. $\mathrm{Li}$, and A. E. Willner, "Tunable chromatic dispersion compensation in $40-\mathrm{Gb} / \mathrm{s}$ systems using nonlinearly chirped fiber bragg gratings," $J$. Lightw. Technol., vol. 20, no. 12, pp. 2239-2246, Dec. 2002.

[10] T. Inui, T. Komukai, K. Mori, and T. Morioka, " $160-\mathrm{Gb} / \mathrm{s}$ adaptive dispersion equalization using an asynchronous dispersion-induced chirp monitor," J. Lightw. Technol., vol. 23, no. 6, pp. 2039-2045, Jun. 2005.

[11] S. Wielandy, M. Fishteyn, and B. Zhu, "Optical performance monitoring using nonlinear detection," J. Lightw. Technol., vol. 22, no. 3, pp. 784-793, Mar. 2004.

[12] K. Bondarczuk, P. J. Maguire, L. P. Barry, J. o’Dowd, W. H. Guo, M. Lynch, A. L. Bradley, J. F. Donegan, and H. Folliot, "Chromatic dispersion monitoring of 80-Gb/s OTDM data signal VIA two-photon absorption in a semiconductor microcavity," IEEE Photon. Technol. Lett., vol. 19, no. 1, pp. 21-23, Jan. 2007.

[13] W. Kaiser and C. G. B. Garrett, "Two photon excitation in CaF2:Eu2+,"Phys. Rev. Lett., vol. 7, no. 6, pp. 229-231, 1961.
[14] H. P. Weber, "Two-photon-absorption laws for coherent and incoherent radiation," IEEE J. Quantum Electron, vol. 7, pp. 189-195, 1971.

[15] F. R. Laughton, J. H. Marsh, D. A. Barrow, and E. L. Portnoi, "The twophoton absorption semiconductor waveguide autocorrelator," IEEE J. Quantum Electron, vol. 30, no. 3, pp. 838-845, 1994.

[16] K. Kikuchi, "Highly sensitive interferometric autocorrelator using $\mathrm{Si}$ avalanche photodiode as two-photon absorber," Electron. Lett., vol. 34, no. 1, pp. 123-125, 1998.

[17] H. Folliot, M. Lynch, A. L. Bradley, L. A. Dunbar, J. Hegarty, J. F. Donegan, L. P. Barry, J. S. Roberts, and G. Hill, "Two-photon absorption photocurrent enhancement in bulk $\mathrm{AlGaAs}$ semiconductor microcavities," Appl. Phys. Lett., vol. 80, no. 8, pp. 1328-1330, Feb. 2002.

[18] P. J. Maguire, L. P. Barry, T. Krug, J. O'Dowd, M. Lynch, A. L. Bradley, J. F. Donegan, and H. Folliot, "Highly-efficient optical sampling of a $100 \mathrm{Gbit} / \mathrm{s}$ OTDM data signal VIA two-photon absorption in semiconductor microcavity," Proc. ECOC 2005, no. We3.5.4., pp. 483-484, 2005.

[19] T. Krug, W. H. Guo, J. O'Dowd, M. Lynch, A. L. Bradley, J. F. Donegan, P. J. Maguire, L. P. Barry, and H. Folliot, "Resonance tuning of two-photon absorption microcavities for wavelength-selective pulse monitoring," IEEE Photon. Technol. Lett., vol. 18, no. 2, pp. 433-435, Jan. 2006.

[20] M. D. Dvorak, W. A. Schroeder, D. R. Andersen, A. L. Smirl, and B. S. Wherrett, "Measurement of the anisotropy of two-photon absorption coefficients in zincblende semiconductors," IEEE J. Quantum Electron, vol. 30, no. 2, pp. 256-268, 1994.

[21] W. H. Guo, J. O’Dowd, M. Lynch, A. L. Bradley, J. F. Donegan, and L. P. Barry, "Influence of cavity lifetime on high-finesse microcavity two-photon absorption photodetectors," IEEE Photon. Technol. Lett., vol. 19, no. 6, pp. 432-434, Mar. 2007.

[22] D. Marcuse, "Pulse distortion in single-mode fibers," Appl. Opt., vol. 19, p. $1653,1980$.

[23] G. P. Agrawal, Nonlinear Fiber Optics, 4th ed. Boston, MA: Academic, 2007, ch. 3.

[24] J. Azana and M. A. Muriel, "Technique for multiplying the repetition rates of periodic trains of pulses by means of a temporal selfimaging effect in chirped fiber gratings," Opt. Lett., vol. 24, no. 23, pp. 1672-1674, 1999.

[25] D. Pudo, M. Depa, and L. R. Chen, "Single and multiwavelength alloptical clock recovery in single-mode fiber using the temporal talbot effect," J. Lightw. Technol., vol. 25, no. 10, pp. 2898-2903, Oct. 2007.

K. Bondarczuk received the M.Sc. degree in applied physics and optoelectronics from the Warsaw University of Technology, Warsaw, Poland, in 2004. His dissertation was entitled "Investigation of light propagation in microstructured optical fibers." His research focused on a tunable photonic bandgap based light propagation within photonic crystal fibers (PCF) filed with nematic liquid crystals. One aspect of the research was concerned on the use of highly birefringence PCF's for tunable PMD compensation. He is currently working toward the Ph.D. degree in electronic engineering at Dublin City University (DCU), Ireland.

His research interests are optical signal processing via two-photon absorption in a semiconductor microcavity.

P. J. Maguire was born in Dublin, Ireland, in 1978. He received B.Eng. degree in electronic engineering (first class hons.) from the Dublin City University (DCU), Dublin, Ireland, in 2001 and the Ph.D. degree in 2006 with a thesis entitled "Optical Pulse Generation and Signal Processing for the Development of High-Speed OTDM Networks".

$\mathrm{He}$ is a Postdoctoral Researcher in the Radio and Optical Communications Laboratory, DCU, working in the areas of optical signal processing via twophoton absorption in a semiconductor microcavity, high-speed optical regeneration using SOA's and saturable absorbers, and optical clock recovery in modelocked laser diodes.

D. Reid received the B.Tech. degree in optoelectronics, the M.Sc. degree in physics, and the Ph.D. degree in physics from the University of Auckland, Auckland, New Zealand, in 2000, 2002, and 2007, respectively. His dissertation was entitled "Characterisation of Optical Communication Pulses."

$\mathrm{He}$ is currently working as Post-Doctoral Researcher in the Radio and Optical Communications Groups at Dublin City University, Ireland. His research interests include the development of techniques for the characterization of ultrashort 
optical pulses, in particular, the use of FROG and other linear spectrographic techniques for real-time measurement of optical communication pulses. Other areas of interest include the design of optimized pulse sources and compressors, the latter being made possible by accurate pulse characterization.

L. P. Barry received the B.E. degree in electronic engineering and the M.Eng.Sc. degree in optical communications from University College Dublin, Dublin, Ireland, in 1991 and 1993, respectively. From February 1993 to January 1996 he was a Research Engineer in the Optical Systems Department of France Telecom's Research Laboratories (CNET), Lannion, France. During this period his research involved the use of ultrashort optical pulses in high capacity optical networks, and as a result of this work he received the Ph.D. degree from the University of Rennes, Rennes, France.

In February 1996 he joined the Applied Optics Centre in Auckland University, New Zealand, as a Research Fellow. His work in New Zealand was mainly concerned with optical pulse generation and measurement, and the use of optical nonlinearities for high speed all-optical switching in fibre networks. In March 1998, he took up a lecturing position in the School of Electronic Engineering at Dublin City University, where he has since developed the Radio and Optical Communications Laboratory.

J. O'Dowd was born in Sligo, Ireland on May 27th, 1981. He received the B.A.mod degree in physics from the University of Dublin Trinity College, Dublin, Ireland in 2004, where he is currently working towards the Ph.D.degree in physics.

His research interests include use of two photon absorption for optical performance monitoring of transparent networks.

W. H. Guo was born in Hubei Province, China, in 1976. He received the B.Sc. degree in physics from Nanjing University, Nanjing, China, in 1998, and the $\mathrm{Ph}$.D. degree from the Institute of Semiconductors, Chinese Academy of Sciences, Beijing, China, in 2004, with a thesis on studying the FDTD simulation and fabrication of optical microcavities and semiconductor optical amplifiers.
Since September 2004, he has been a Postdoctoral Researcher in the Department of Physics, Trinity College Dublin, Ireland.

M. Lynch received the B.Sc. degree in applied physics from Dublin City University (DCU), Dublin, Ireland, in 1986.

He is currently a Senior Researcher with the Semiconductor Photonics Group, based in the Department of Physics, University of Dublin, Trinity College. Current research interests include nonlinear optics in semiconductor microcavities and laser diode gas sensin.

A. L. Bradley received the B.Sc. degree in physics from University College Dublin, Dublin, Ireland, in 1992 and the M.Sc. and Ph.D. degrees in physics from Trinity College, Dublin, in 1994 and 1998, respectively.

She was a Postdoctoral Researcher in Trinity College, and a lecturer in the Institute of Technology, Tallaght, Ireland, before taking up her current position as a lecturer in the School of Physics, Trinity College Dublin in 2001. Her curren research interests include microcavity physics and applications, the dynamical properties of semiconductor optical amplifiers, semiconductor nanocrystal dynamics and novel wide bandgap materials.

J. F. Donegan (M'05) received the B.Sc. and Ph.D. degrees from the National University of Ireland, Galway.

He held postdoctoral positions at Lehigh University, Bethlehem, PA, and Max Planck Institut fuer Festkoerperforschung, Stuttgart. He is presently Professor of Physics, Trinity College Dublin, Ireland. He has 120 publications. His research area is photonics with the following subthemes: quantum dot cellular interactions for biomedical applications; nonlinear microcavity photodetectors for optical performance monitoring; tunable diode lasers based on the slotted Fabry Perot cavity; optical sensing applications with tunable diodes; and microsphere and microtube structures for microlaser and microsensor development. 